\title{
Unsteady flow and heat transfer in a thin film of Ostwald-de Waele liquid over a stretching surface
}

\author{
K. Vajravelu ${ }^{1}$, K. V. Prasad ${ }^{2}$ and Chiu-On $\mathrm{Ng}^{3^{*}}$ \\ ${ }^{1}$ Department of Mathematics, University of Central Florida, Orlando, Florida 32816, USA \\ ${ }^{2}$ Department of Mathematics, Bangalore University, Bangalore 560001, India \\ ${ }^{3}$ Department of Mechanical Engineering, The University of Hong Kong, Hong Kong, People’s Republic of China \\ *Corresponding author. E-mail address: cong@hku.hk
}

\begin{abstract}
In this paper, the effects of viscous dissipation and the temperature-dependent thermal conductivity on an unsteady flow and heat transfer in a thin liquid film of a non-Newtonian Ostwald-de Waele fluid over a horizontal porous stretching surface is studied. Using a similarity transformation, the time- dependent boundary-layer equations are reduced to a set of non-linear ordinary differential equations. The resulting five parameter problem is solved by the Keller-Box method. The effects of the unsteady parameter on the film thickness are explored numerically for different values of the power-law index parameter and the injection parameter. Numerical results for the velocity, the temperature, the skin friction and the wall-temperature gradient are presented through graphs and tables for different values of the pertinent parameter. One of the important findings of the study is that the film thickness increases with an increase in the powerlaw index parameter (as well as the injection parameter). Quite the opposite is true with the unsteady parameter. Furthermore, the wall-temperature gradient decreases with an increase in the Eckert number or the variable thermal conductivity parameter. Furthermore, the surface temperature of a shear thinning fluid is larger compared to the Newtonian and shear thickening fluids. The results obtained reveal many interesting behaviors that warrant further study of the equations related to non-Newtonian fluid phenomena, especially the shear-thinning phenomena.
\end{abstract}

Keywords: Thin film flow, heat transfer, variable fluid property, power-law fluid, viscous dissipation, numerical method.

\section{Introduction}

During the past two decades, due to its applications to several areas in science and engineering, considerable attention has been devoted to the study of flow and heat transfer within a thin liquid 
film on an unsteady stretching sheet. These areas include extrusion processes, wire and fiber coating, polymer processing, food stuff processing, design of various heat exchangers and chemical processing equipment, etc. In particular, in melt-spinning processes, the extrudate from the die is generally drawn and simultaneously stretched into a filament or sheet, which is then solidified through rapid quenching or gradual cooling by direct contact with water or chilled metal rolls. In fact, stretching imparts a unidirectional orientation to the extrudate and, as a consequence, the quality of the final product depends considerably on the flow and heat transfer mechanism. Therefore, the analysis of momentum and thermal transport within a thin liquid film on a continuously stretching surface is important for gaining some fundamental understanding of such processes. Motivated by the process of polymer extrusion, in which the extrudate emerges from a narrow slit, Crane [1] examined the Newtonian fluid flow induced by the stretching of an elastic flat sheet. Subsequently, several extensions related to Crane's [1] flow problem were made for different physical situations (see [2-6]). In these studies [1-6], the boundary layer equation is considered and the boundary conditions are prescribed at the sheet and on the fluid at infinity. Imposition of a similarity transformation reduced the system to a set of ordinary differential equations (ODEs), which was then solved analytically or numerically.

All the above mentioned studies deal with flow and/or heat transfer from a stretching sheet in a fluid medium extending to infinity. However, in real physical situations involving coating processes, one needs to consider the fluid adhering to the stretching sheet as a finite liquid film. Wang [7] was the first to consider such a flow problem with a finite liquid film of a Newtonian fluid over an unsteady stretching sheet. Later, Usha and Sridharan [8] considered a similar problem of axi-symmetric flow in a liquid film. Dandapat et al. [9] investigated the effects of variable fluid properties and thermo-capillarity on the flow and heat transfer in a liquid film on a horizontal stretching sheet. Further, Liu and Andersson [10] explored the work of [7] to study the thermal characteristics of liquid film on an unsteady stretching surface. Abel et al. [11] studied the heat transfer problem for a thin liquid film in the presence of an external magnetic field with viscous dissipation. Nadeem and Awais [12] analyzed the effect of a thin film flow over an unsteady shrinking sheet with variable viscosity. Recently, Aziz et al. [13] addressed the influence of internal heat generation/absorption on the flow and heat transfer in a thin film on an unsteady stretching sheet. 
It should be noted that the flow and heat transfer characteristics are affected not only by the velocity and the thermal boundary conditions but also by the physical properties of the liquidfilm. Furthermore, the study of non-Newtonian fluid flow on an unsteady stretching surface is important. Although the fluid employed in material processing or protective castings are generally non-Newtonian (example, most of the paints), there has been a very little work done on the flow and heat transfer of a non-Newtonian liquid film over a stretching surface. Among the most popular rheological models of non-Newtonian fluids is the power-law or Ostwald-deWaele model. This model deals with a simple non-linear equation of state for inelastic fluids; this includes linear Newton-fluids as a special case. The power- law model provides an adequate representation of many non-Newtonian fluids for range of shear rates. For instance, Andersson et al. [14] carried out a numerical study for the hydro-dynamical problem of a power-law fluid flow with in a liquid film over a stretching sheet. Here, the thermo-physical properties of the ambient fluid are assumed to be constant. However, it is well known that these properties may change with temperature, especially the thermal conductivity. Available literature [15-17] on variable thermal conductivity shows that this type of work has not been carried out for non-Newtonian fluid obeying the Ostwald-de Waele power-law model.

The purpose of the present study is to explore the effects of thermo-physical property, namely, the variable thermal conductivity and the viscous dissipation on the heat transfer of an incompressible power-law liquid thin film on an unsteady porous stretching surface. In nonNewtonian liquid thin film flow, the effects of variable thermal conductivity, power law index, and viscous dissipation play a significant role in the heat transfer process. Here, the momentum and energy equations are highly non-linear. Hence, a similarity transformation is used to transform the non-linear partial differential equations into nonlinear ordinary differential equations. Due to its complexity and nonlinearity, the proposed problem, is solved numerically by a finite difference scheme known as the Keller box method. The obtained numerical results are used to analyze the flow and heat transfer characteristics of the power-law liquid film that would find applications in manufacturing industries. 


\section{Formulation of the mathematical problem}

Consider an unsteady, two-dimensional, viscous, laminar flow and heat transfer of an incompressible non-Newtonian thin fluid film obeying a power-law model. The flow is due to the stretching of a porous elastic sheet parallel to the $x$-axis at $y=0$. Two equal and opposite forces are applied along the $x$-axis, keeping the origin fixed. A schematic representation of the physical model is presented in figure 1. The continuous stretching sheet is assumed to have a prescribed velocity $U_{s}(x, t)$ and temperature $T_{s}(x, t)$. Further, a thin liquid film of uniform thickness $h(t)$ rests on the horizontal sheet. With the above assumptions, the equations of conservation of mass, momentum, and energy can be written as

$$
\begin{aligned}
& \frac{\partial u}{\partial x}+\frac{\partial \mathrm{v}}{\partial y}=0 \\
& \frac{\partial u}{\partial t}+u \frac{\partial u}{\partial x}+\mathrm{v} \frac{\partial u}{\partial y}=\frac{1}{\rho} \frac{\partial \tau_{x y}}{\partial y}, \\
& \rho c_{p}\left(\frac{\partial T}{\partial t}+u \frac{\partial T}{\partial x}+\mathrm{v} \frac{\partial T}{\partial y}\right)=\frac{\partial}{\partial y}\left(\kappa(T) \frac{\partial T}{\partial y}\right)+K\left(\frac{\partial u}{\partial y}\right)^{n+1},
\end{aligned}
$$

where $u$ and $\mathrm{v}$ are the velocity components along the $x$ and $y$ directions, respectively; $\rho$ is the density, $\tau_{x y}$ is the shear stress. Here, we assume the shear stress as

$$
\tau_{x y}=-K\left(-\frac{\partial u}{\partial y}\right)^{n}
$$

where $K$ is the consistency coefficient and $n$ is the flow behavior index, namely, the power-law index. The fluid is Newtonian for $n=1$ with $K=\mu$ (the absolute viscosity). As $n$ deviates from unity, the fluid becomes non-Newtonian: For example, $n<1$ and $n>1$ correspond to shear thinning (pseudo plastic) and shear thickening (dilatants) fluids, respectively. Further, $C_{p}$ is the specific heat at constant pressure, $T$ is the temperature, and $\kappa(T)$ is the temperature-dependent variable thermal conductivity. For liquid metals, the thermal conductivity varies linearly with temperature in the range $0^{0} \mathrm{~F}$ to $400^{\circ} \mathrm{F}$ (see for details Savvas et al. [15]). In the present study, the thermal conductivity is assumed to vary linearly with temperature (Chaim [23]) as 


$$
\kappa(T)=\kappa_{0}\left(1+\frac{\varepsilon}{\Delta T}\left(T-T_{0}\right)\right)
$$

Here, $\Delta T=\left(T_{s}-T_{0}\right), T_{s}$ is the temperature of the stretching sheet, $\varepsilon$ is a small parameter known as the variable thermal conductivity parameter, and $\kappa_{0}$ is the thermal conductivity of the fluid. The last term in equation (2.3) is due to the viscous dissipation.

Substituting (2.4)-(2.5) into equations (2.2)-(2.3) we obtain

$$
\begin{aligned}
& \frac{\partial u}{\partial t}+u \frac{\partial u}{\partial x}+\mathrm{v} \frac{\partial u}{\partial y}=-\frac{K}{\rho} \frac{\partial}{\partial y}\left(-\frac{\partial u}{\partial y}\right)^{n}, \\
& \rho c_{p}\left(\frac{\partial T}{\partial t}+u \frac{\partial T}{\partial x}+\mathrm{v} \frac{\partial T}{\partial y}\right)=\frac{\partial}{\partial y}\left(\kappa_{0}\left(1+\varepsilon \frac{T-T_{0}}{\Delta T}\right) \frac{\partial T}{\partial y}\right)+K\left(\frac{\partial u}{\partial y}\right)^{n+1} .
\end{aligned}
$$

In the derivation of the above governing equations, the conventional boundary layer approximation has been invoked. This is justified by the assumption that the film thickness $h$ is much smaller than the characteristic length $L$ (in the direction along the sheet). The mass conservation equation (2.1) then implies that the ratio $(\mathrm{v} / u)$ between the two velocity components is of order $\left(h / L^{2}\right)$. Also, streamwise diffusion of momentum and thermal energy is of order $\left(h / L^{2}\right)$, smaller than the corresponding diffusion perpendicular to the sheet. For this reason the stream wise diffusion terms are neglected in equations (2.6) and (2.7).

Assuming that the interface of the planar liquid film is smooth and free of surface waves and the viscous shear stress and the heat flux vanish at the adiabatic free surface, the boundary conditions become

$$
\begin{aligned}
& u=U_{s} \quad \mathrm{v}=\mathrm{v}_{s} \quad T=T_{s} \quad \text { at } \quad y=0, \\
& \frac{\partial u}{\partial y}=\frac{\partial T}{\partial y}=0, \quad \mathrm{v}=\frac{d h}{d t} \quad \text { at } y \rightarrow h(t),
\end{aligned}
$$

where $U_{s}$ and $T_{s}$ are the surface velocity and temperature of the stretching sheet, respectively, and $\mathrm{v}_{s}$ is the injection parameter. Here $h(t)$ is the free surface elevation of the liquid film. That 
is, the film thickness. In this paper, the flow is caused by the linear stretching of the elastic sheet at $y=0$ with a velocity of the form

$$
U_{s}=\frac{b x}{1-\alpha t}
$$

where $b$ and $\alpha$ are both positive constants with dimension reciprocal of time $t$. Here $b$ is the initial stretching rate, whereas $\frac{b}{1-\alpha t}$ is the effective stretching. In the context of polymer extrusion, the material properties, in particular the elasticity of the extruded sheet, may vary with time even though the sheet is being pulled by a constant force. The dimensionless ratio $S \cong \alpha / b$, is the only parameter in Wang's [7] analysis; in the limiting case as $S \rightarrow 0$, Wang's case reduces to the steady-state problem of Crane [1]. With unsteady stretching $(\alpha \neq 0)$, however, $\alpha^{-1}$ becomes the representative time scale of the resulting unsteady boundary layer problem. The adopted formulation of the sheet velocity $U_{s}(x, t)$ in equation (2.10) is valid for $t<\alpha^{-1}$ only, unless $\alpha=0$. Further, it should be noted that the end effects and the gravity are negligible, and the surface tension is sufficiently large such that the film surface remains smooth and stable throughout the motion.

The surface temperature $T_{s}$ of the sheet varies with the distance $x$ from the slot and time $t$ : $T_{s}=T_{0}-T_{\text {ref }}\left[\frac{b^{2-n} x^{2}}{2(K / \rho)}\right](1-\alpha t)^{n-5 / 2}$,

where $T_{0}$ is the fixed temperature at the slit, and $T_{\text {ref }}$ is the reference temperature, which can be taken as $T_{\text {ref }}=T_{0}$ in the present study. The constant of proportionality $d$ is assumed to be positive with dimension (time ${ }^{-1}$ ). Equation (2.11) represents a situation in which the sheet temperature decreases from $T_{0}$ and is proportion to $x^{2}$, and the amount of temperature reduction along the sheet increases with time. It should be noted that the expressions given by equations (2.10) and (2.11) are valid for time $t<(1 / \alpha)$. The assumptions about $U_{s}$ and $T_{s}$ in equations (2.10) and (2.11) respectively allow us to develop a similarity transformation which converts the partial 
differential equations (PDEs) into a set of ODEs. We introduce the following dimensionless variable $f(\xi)$ and $\theta(\xi)$ as well as the similarity variable $\xi$ thus:

$$
\begin{aligned}
& \psi=\left(\frac{b^{1-2 n}}{(K / \rho)}\right)^{\frac{1}{n+1}} x^{\frac{2 n}{n+1}}(1-\alpha t)^{\frac{1-2 n}{n+1}} \beta^{-1} f(\xi), \\
& T=T_{0}-T_{r e f}\left[\frac{b^{2-n} x^{2}}{2(K / \rho)}\right](1-\alpha t)^{n-\frac{5}{2}} \theta(\xi), \\
& \xi=\left[\frac{b^{2-n} x^{2}}{(K / \rho)}\right] x^{(1-n) /(1+n)}(1-\alpha t)^{(n-2) /(n+1)} \beta^{-1} y .
\end{aligned}
$$

In equation (2.12) the stream function $\psi(x, y, t)$ is defined by $u=\partial \psi / \partial y$ and $v=-\partial \psi / \partial x$, such that the continuity equation (2.1) is satisfied automatically, and $\beta$ is a constant denoting the dimensionless film thickness. In terms of these new variables, the momentum and the energy equations together with the boundary conditions become

$$
\begin{aligned}
& n\left(-f^{\prime \prime}\right)^{n-1} f^{\prime \prime \prime}+\beta^{n+1}\left(\frac{2 n}{n+1} f f^{\prime \prime}-f^{\prime 2}\right)-S \beta^{n+1}\left(f^{\prime}+\frac{2-n}{n+1} \xi f^{\prime \prime}\right)=0 \\
& \frac{1}{\operatorname{Pr}}\left((1+\varepsilon \theta) \theta^{\prime}\right)^{\prime}+\beta^{2}\left|\begin{array}{cc}
\frac{2 n}{n+1} f & f^{\prime} \\
2 \theta & \theta^{\prime}
\end{array}\right|-S \beta^{2}\left[\left(\frac{5}{2}-n\right) \theta-\left(\frac{2-n}{n+1}\right) \xi \theta^{\prime}\right]+E c \beta^{1-n}\left(f^{\prime \prime}\right)^{n+1}=0
\end{aligned}
$$

and

$$
\begin{aligned}
& f(0)=f_{w}, \quad f^{\prime}(0)=1, \quad f^{\prime \prime}(1)=0, \quad f(1)=\left(\frac{2-n}{2 n}\right) S, \\
& \theta(0)=1, \quad \theta^{\prime}(1)=0,
\end{aligned}
$$

where a prime denotes the differentiation with respect to $\xi, S=\alpha / b$ is a dimensionless measure of the unsteadiness, $\operatorname{Pr}=\left(U_{s} / \alpha\right) \operatorname{Re}_{x}{ }^{\frac{-2}{n+1}}$ is the generalized Prandtl number, $f_{w}=-\left(\mathrm{v}_{s} / U_{s}\right)(2 n / n+1) \operatorname{Re}_{x}{ }^{\frac{1}{n+1}}$ is the suction/injection parameter (namely, $f_{w}>0$ corresponds to suction whereas $f_{w}<0$ corresponds to injection), and $E c=U_{s}^{2} / C_{p}\left(T_{s}-T_{0}\right)$ is the Eckert number. The parameter $\beta$ is an unknown constant which must be determined as a part of the boundary value problem. 
Although the dimensionless film thickness is a constant for fixed values of $S$ and $n$, the actual film thickness depends on time $t$ and the streamwise location $x$. From equation (2.14) we find that the film thickness $h(x, t)$ can be expressed as

$$
h(x, t)=\beta\left(\frac{(K / \rho)}{b^{2-n}}\right) x^{(n-1) /(n+1)}(1-\alpha t)^{(2-n) /(1+n)}
$$

In the Newtonian case $(n=1), h$ becomes a function of time only; whereas for non-Newtonian films, the thickness decreases with $x$ for pseudo plastics $(n<1)$, while the film thickness decreases in the streamwise direction for dilatant fluids $(n>1)$.

It is worth mentioning here that the momentum boundary layer problem defined by the ODE (2.15) subject to the relevant boundary conditions (2.17) is de-coupled from the thermal boundary layer problem, while the temperature field is on the other hand coupled with the velocity field. For practical purposes, the physical quantities of interest include the velocity components $u$ and $\mathrm{v}$, the local skin friction coefficient $C_{f x}$, and the local Nusselt number $N u_{x}$. These quantities can be written as

$$
\begin{aligned}
& u=U_{s} f^{\prime}, \\
& \mathrm{v}=-U_{s} \operatorname{Re}_{x}^{-1 /(n+1)}\left(\frac{2 n}{n+1} f+\frac{1-n}{1+n} \xi f^{\prime}\right), \\
& C_{f x}=2 \operatorname{Re}_{x}^{-1 /(n+1)}\left[-f^{\prime \prime}(0)\right]^{n}, \\
& N u_{x}=\frac{1}{2}(1-\alpha t)^{\frac{-1}{2}} \operatorname{Re}_{x}^{\frac{n+2}{n+1}} \theta^{\prime}(0),
\end{aligned}
$$

where $\operatorname{Re}_{x}=\rho U_{s}^{2-n} x^{n} / K$ is the local Reynolds number.

\section{Numerical procedure}

The system of equations (2.15) and (2.16) are highly non-linear ordinary differential equations of third-order and second-order, respectively. Exact analytical solutions are not possible for the complete set of equations (2.15) and (2.16). Hence, we use the efficient numerical method with second order finite difference scheme known as the Keller-Box method [18-19]. This method is 
unconditionally stable and has a second order accuracy with arbitrary spacing. First, we write the differential equations and the boundary conditions in terms of first order system, which is then converted to a set of finite difference equations using central differences. Then the non-linear algebraic equations are linearized by Newton's method and the resulting linear system of equations is solved by block tri-diagonal elimination technique. For the sake of brevity, the details of the solution process are not presented here. For numerical calculations, a uniform step size of $\Delta \xi=0.005$ is found to be satisfactory and the solutions are obtained with an error tolerance of $10^{-6}$ in all the cases. To demonstrate the accuracy of the present method, results for the dimensionless film thickness and the skin friction are compared with the available results in the literature for a special case: That is, for a Newtonian fluid ( $n=1)$, obtained by Aziz et al. [13] and Wang [7]. It was found from Table 1 that the present results agree very well with those of Aziz et al. [13] and Wang [7].

\section{Results and discussion}

In order to analyze the effects of the pertinent parameters, namely, the power-law index $n$, the dimensionless film thickness $\beta$, the unsteady parameter $S$, the injection parameter $f_{w}$, the variable thermal conductivity parameter $\varepsilon$, the modified Prandtl number Pr, and the Eckert number $E c$ on the flow and heat transfer characteristics, the numerical solutions are obtained. Also, in order to get a clear insight in to the physical problem, the velocity and the temperature fields are presented graphically in figures 2-7. Values of the skin friction, the dimensionless film thickness and the wall-temperature gradient for different values of the physical parameters are recorded in Tables 2 and 3. For this hydrodynamic problem, there exists a critical value of $S$, above which no solution could be obtained: Wang [7] noticed the critical value of $S=2$ for Newtonian fluid. It may be noted here that (for positive values of $S$ ), $S \rightarrow 0$ stands for the case of an infinitely thick fluid layer (i.e., $\beta \rightarrow \infty$ ), whereas the limiting case of $S \rightarrow 2$ represents a liquid film of infinitesimal thickness (i.e., $\beta \rightarrow 0$ ). In the case of non-Newtonian fluids, the present calculations show that the critical value of $S=1.35$ for shear thinning fluids and the critical value of $S=3.03$ for shear thickening fluids when $\beta \rightarrow 0$. However, it is difficult to perform these calculations for the limiting case of $\beta \rightarrow \infty$. 
The transverse velocity profiles $(f)$ and the horizontal velocity profiles $\left(f^{\prime}\right)$ for blowing and suction cases are shown graphically in figures 2-3 with different values of $S, f_{w}$ and $n$. The general trend is that $f^{\prime}$ decreases monotonically, whereas $f$ increases monotonically as the distance increases from stretching sheet. The effect of increasing values of $S$ is to increase $f$ and $f^{\prime}$ and thereby reduce the horizontal boundary layer thickness. This phenomenon is true even for shear thinning ( $n=0.8)$, Newtonian $(n=1)$ and shear thickening $(n=1.2)$ fluids. We further notice from these figures that a moderate deviation from Newtonian rheology $(n=1)$ have a significant influence on the horizontal velocity component $f^{\prime}$ across the fluid film. For a given value of $S$, the pseudo plastic (shear thinning fluids) film is thinner and exhibits a greater surface velocity than a Newtonian film, while quite reverse behavior is true for shear thickening (dilatant) fluids. In shear thinning fluids, viscosity is reduced with increasing shear rates; whereas for dilatants substances, viscosity increases with shear rate and becomes more viscous and will thicken with an increasing rate of shear. It is therefore not surprising to observe that the pseudo plastics are more likely to flow nearly as an inviscid layer on top of the stretching sheet than as in the case of shear thickening or dilatants fluids. These results are in good agreement with the physical situations. Comparison of figure 3(a) with figure 3(b) reveals that suction $\left(f_{w}>0\right)$ reduces the horizontal velocity boundary layer thickness whereas blowing $\left(f_{w}<0\right)$ has quite the opposite effect on the velocity boundary layer.

The effects of the power law index parameter on the temperature profiles for $f_{w}<0$ and $f_{w}>0$ are shown graphically in figures 4(a)-4(b). It is observed that the temperature distribution is unity at the wall. With changes in the physical parameters it decreases as the distance increases from the sheet. Further, the effect of increasing $\mathrm{S}$ with different values of $n$, [namely, shear thinning ( $n$ $=0.8)$, Newtonian ( $n=1)$, and shear thickening $(n=1.2)$ fluids] is to reduce the temperature, and hence the thermal boundary layer thickness. Comparison of figure 4(a) with figure 4(b) reveals that the effect of the injection parameter is to reduce the thermal boundary layer thickness. Figures 5(a) and 5(b) exhibit the temperature distribution $\theta(\xi)$ with $\xi$ for different values of $E c$ in blowing and suction cases, respectively. From these figures we see that the effect of increasing 
$E c$ is to increase the temperature distribution $\theta(\xi)$. This is in conformity with the fact that energy is stored in the fluid region as a consequence of dissipation due to viscosity and elastic deformation.

The effects of $\varepsilon$ on the temperature profile in the boundary layer for both $f_{w}<0$ and $f_{w}>0$ are depicted in figures 6(a) and 6(b), respectively. From these figures, we observe that the temperature distribution is lower throughout the boundary layer for zero values of $\varepsilon$ as compared with non-zero values of $\varepsilon$. This is due to the fact that the presence of temperature-dependent thermal conductivity results in reducing the magnitude of the transverse velocity by a quantity $\partial K(T) / \partial y$, and this can be seen from the energy equation. This behavior holds for all types of fluids considered, namely, pseudo plastic, Newtonian, and dilatant fluids. The variations of temperature profile $\theta(\xi)$ with $\xi$ for various values of the modified Prandtl number $\operatorname{Pr}$ are shown in figures 7(a) and 7(b) for both $f_{w}<0$ and $f_{w}>0$, respectively. Both figures demonstrate that an increase in $\operatorname{Pr}$ results in a monotonic decrease in the temperature distribution and it tends to zero as the distance increases from the sheet. That is, the thermal boundary layer thickness decreases for higher values of the Prandtl number. This holds good for all values of $n$ and $f_{w}$.

The values of $f^{\prime \prime}(0), \theta^{\prime}(0), S$, and $f_{w}$ are recorded in Table 2 . It is interesting to note that $\beta$, $\left|f^{\prime \prime}(0)\right|$ and $\left|\theta^{\prime}(0)\right|$ decrease gradually with increasing S. This is true for all values of $n$. Further, the effect of increasing $n$ and $f_{w}$ is to enhance $\beta,\left|f^{\prime \prime}(0)\right|$ and $\left|\theta^{\prime}(0)\right|$. From Table 3, we see that the effect of $E c$ and $\varepsilon$ is to decrease the magnitude of the wall- temperature gradient; whereas the effect of $\operatorname{Pr}$ is to enhance it. This is true for all values of $n$ and $f_{w}$.

\section{Conclusions}

The purpose of the present work is to obtain numerical solutions to the problem of flow and heat transfer in a power-law liquid film on an unsteady porous stretching sheet in the presence of viscous dissipation and temperature-dependent thermal conductivity. Results for the velocity and the temperature distributions across the liquid film, the free surface velocity $f^{\prime}(1)$, the 
temperature $\theta(1)$, the wall-shear stress, and the wall-temperature gradient are presented for different values of the governing parameters. The results obtained might be useful for the material processing industries. We summarize some of the interesting results below:

1. In comparison with the Newtonian fluid, the free surface temperature is enhanced for shear thinning fluid, while it is decreased for shear thickening fluid. Also free surface temperature approaches zero for higher values of the Prandtl number.

2. The effect of suction is to reduce the thermal boundary layer thickness as compared to blowing. This holds for all values of the power-law index, the variable thermal conductivity and the Eckert number.

3. The effect of viscous dissipation is found to increase the dimensionless free surface temperature $\theta(1)$. This observation is true for all values of the power-law index.

Acknowledgments: The authors appreciate the constructive comments of the reviewers which led to definite improvements in the paper. The paper was completed when KV was visiting the University of Hong Kong in July, 2011. The work was partially supported by the Research Grants Council of the Hong Kong Special Administrative Region, China, through Project No. HKU 715510E.

\section{References}

1. L.J. Crane, Flow past a stretching plate, ZAMP 21(1970) 645-647.

2. B. Siddappa and M. Subhas Abel, `Non-Newtonian flow past a stretching plate, ZAMP 36 (1985) 890-892.

3. B.S. Dandapat, A.S. Gupta, Flow and heat transfer in a visco-elastic fluid over a stretching sheet, Int. J. Non-Linear Mech. 24 (1989) 241-219.

4. R. Cortell, Similarity solutions for flow and heat transfer of a viscoelastic fluid over a stretching sheet, Int. J. Non-Linear Mech. 29 (1994) 155-161. 
5. I. A. Hassanien, A. A. Abdullah, R. S. R. Gorla, Flow and heat transfer in a power-law fluid over a non isothermal stretching sheet, Math. Comp. Model. 28 (1998) 105-116.

6. M.E. Ali, On the thermal boundary layer on a power law stretched surface with suction or injection, Int. J. Heat and Fluid flow, 16 (1995) 280-290.

7. C.Y. Wang, Liquid film on an unsteady stretching surface, Quart. Appl. Math. 48 (1990) 601-610.

8. R.Usha, R.Sridharan, on the motion of a liquid film on an unsteady stretching surface, ASME Fluids Eng. 150 (1993) 43-48.

9. B.S.Dandapat, B.Santra, K.Vajravelu, The effects of variable fluid properties and thermocapillarity on the flow of a thin film on an unsteady stretching sheet, Int. J. Heat Mass Transfer. 50 (2007) 991-996.

10. I.C.Liu, H.I.Andersson, Heat transfer on an unsteady stretching sheet, Int. J. Thermal Sci. 47 (2008) 766-772.

11. M.S. Abel, N. Mahesha, J. Tawade, Heat transfer in a liquid film on an unsteady stretching surface with viscous dissipation in the presence of external magnetic field, Appl. Math. Model. 33 (2009) 3430-3441.

12. S. Nadeem, M. Awais, Thin film flow of an unsteady shrinking sheet, through medium with variable viscosity, Phys Letters A. 372 (2008) 4695-4972.

13. R. C. Aziz, I. Hasim, A. K. Almari, Thin film flow and heat transfer on an unsteady stretching sheet with internal heating, Meccanica. 46 (2011) 349-357.

14. H.I. Andersson, J. B. Aaresh, N. Braud, B. S. Dandapat, Flow of a power law fluid on an unsteady stretching surface, J. non-Newtonian Fluid Mech. 62 (1996) 1-8.

15. T. A. Savvas N.C. Markatos, C. D. Papaspyrides, On the flow of non-Newtonian polymer solutions. Appl Math Model. 18 (1994)14-22. 
16. T.C. Chaim, Heat transfer in a fluid with variable thermal conductivity over a linearly stretching sheet, Acta Mech. 129 (1998) 63-72.

17. K. V. Prasad, Dulal Pal and P. S. Datti, MHD flow and heat transfer in the flow of a power law fluid over a non-isothermal stretching sheet, CNSNS 14 (2009) 2178-2189.

18. T. Cebeci, P. Bradshaw, Physical and computational aspects of convective heat transfer. New York: Springer-Verlag; 1984.

19. H.B. Keller, Numerical methods for two-point boundary value problems. New York: Dover Publ.; 1992. 
Table 1: Variation of the dimensionless film thickness and the skin friction with unsteady parameter for $n=1$ when $\operatorname{Pr}=1.0$.

\begin{tabular}{|c|c|c|c|c|c|c|}
\hline \multirow{2}{*}{$s$} & \multicolumn{2}{|c|}{ Present work } & \multicolumn{2}{c|}{ Aziz et al (2011) } & \multicolumn{2}{c|}{ Wang (2006) } \\
\cline { 2 - 7 } & $\beta$ & $f^{\prime \prime}(0)$ & $\beta$ & $f^{\prime \prime}(0)$ & $\beta$ & $f^{\prime \prime}(0)$ \\
\hline 0.8 & 2.149956 & -2.677546 & 2.151994 & -2.680943 & 2.15199 & -2.68094 \\
\hline 1.0 & 1.540905 & -1.967298 & 1.543616 & -1.972384 & 1.54362 & -1.97238 \\
\hline 1.2 & 1.124422 & -1.435752 & 1.127780 & -1.442625 & 1.127780 & -1.442631 \\
\hline 1.4 & 0.816898 & -1.003991 & 0.821032 & -1.012784 & 0.821032 & -1.012784 \\
\hline 1.6 & 0.570868 & -0.631578 & 0.576173 & -0.642397 & 0.567173 & -0.642397 \\
\hline 1.8 & 0.348569 & -0.296197 & 0.356389 & -0.309137 & 0.356389 & -0.309137 \\
\hline
\end{tabular}

Table 2: Values of the dimensionless film thickness, skin friction, and the wall-temperature gradient for different values of the physical parameters when $E c=0.0, \varepsilon=0.0$, and $\operatorname{Pr}=1.0$.

\begin{tabular}{|c|c|c|c|c|c|c|c|c|c|c|}
\hline \multirow{2}{*}{$n$} & \multirow{2}{*}{$s$} & \multicolumn{3}{|c|}{$f_{w}=-0.1$} & \multicolumn{3}{|c|}{$f_{w}=0.0$} & \multicolumn{3}{|c|}{$f_{w}=0.1$} \\
\hline & & $\beta$ & $f^{\prime \prime}(0)$ & $\theta^{\prime}(0)$ & $\beta$ & $f^{\prime \prime}(0)$ & $\theta^{\prime}(0)$ & $\beta$ & $f^{\prime \prime}(0)$ & $\theta^{\prime}(0)$ \\
\hline \multirow{5}{*}{0.8} & 0.4 & 2.946857 & -3.055868 & -4.160374 & 3.807364 & -4.486288 & -5.790560 & 4.935935 & -7.201140 & -8.525701 \\
\hline & 0.6 & 1.684658 & -1.885005 & -2.596097 & 2.156749 & -2.646549 & -3.473627 & 2.732279 & -3.788101 & -4.704508 \\
\hline & 0.8 & 1.016171 & -1.106725 & -1.616648 & 1.322460 & -1.614392 & -2.212826 & 1.680126 & -2.276095 & -2.951423 \\
\hline & 1.0 & 0.572817 & -0.502090 & -0.829930 & 0.811267 & -0.896435 & -1.335308 & 1.065762 & -1.361451 & -1.881660 \\
\hline & 1.2 & 0.250278 & -0.107785 & -0.224396 & 0.423738 & -0.322772 & -0.566811 & 0.648091 & -0.696177 & \\
\hline \multirow{6}{*}{1.0} & 0.4 & 3.814856 & -3.686102 & & 4.981264 & -5.649096 & -7.540908 & 7.112672 & -10.88815 & 392 \\
\hline & 0.6 & 2.451166 & -2.646380 & -3.64 & 3.130509 & -3.740963 & -4.99 & 4.051656 & -5.695822 & \\
\hline & 0.8 & 1.694112 & -1.949764 & -2.693337 & 2.149956 & -2.677546 & -3.592371 & 2.717756 & -3.773035 & -4.894077 \\
\hline & 1.0 & 1.208905 & -1.426557 & -2.002029 & 1.540905 & -1.967298 & -2.671805 & 1.938147 & -2.698758 & -3.553401 \\
\hline & 1.5 & 0.479897 & -0.459440 & -0.707743 & 0.688502 & -0.812051 & -1.185444 & 0.907301 & -1.215614 & -1.697482 \\
\hline & 1.8 & 0.151347 & -0.060926 & -0.100959 & 0.348569 & -0.296197 & -0.465575 & 0.546683 & -0.632880 & -0.938642 \\
\hline \multirow{7}{*}{1.2} & 0.4 & 4.562485 & -4.175917 & -5.900652 & 6.386359 & -7.075603 & -9.588795 & 8.561658 & -13.595714 & -17.478777 \\
\hline & 0.6 & 3.168481 & -3.258797 & -4.514707 & 4.172085 & -4.858248 & -6.572510 & 6.259832 & -9.265298 & -12.06632 \\
\hline & 0.8 & 2.359576 & -2.627898 & -3.607574 & 3.030494 & -3.690320 & -4.985146 & 4.080532 & -5.765680 & -7.616121 \\
\hline & 1.0 & 1.825443 & -2.153828 & -2.948138 & 2.318130 & -2.935676 & -3.964484 & 2.992874 & -4.211517 & -5.600378 \\
\hline & 1.5 & 1.036959 & -1.312976 & -1.818325 & 1.318035 & -1.783379 & -2.429001 & 1.657002 & -2.399008 & -3.224663 \\
\hline & 2.0 & 0.584205 & -0.698526 & -0.992735 & 0.782885 & -1.053527 & -1.469266 & 1.002888 & -1.466741 & -2.008754 \\
\hline & 2.5 & 0.230544 & -0.179074 & -0.248906 & 0.421897 & -0.483644 & -0.689867 & 0.597107 & -0.814618 & -1.147503 \\
\hline
\end{tabular}


Table 3: Values of the wall-temperature gradient for different values of the physical parameters when $s=0.8$.

\begin{tabular}{|c|c|c|c|c|c|c|c|c|c|c|c|}
\hline \multirow[t]{2}{*}{$\operatorname{Pr}$} & \multirow[t]{2}{*}{ Ec } & \multirow[t]{2}{*}{$\varepsilon$} & \multicolumn{3}{|c|}{$n=0.8$} & \multicolumn{3}{|c|}{$n=1.0$} & \multicolumn{3}{|c|}{$n=1.2$} \\
\hline & & & $f_{w}=-0.1$ & $f_{w}=0.0$ & $f_{w}=0.1$ & $f_{w}=-0.1$ & $f_{w}=0.0$ & $f_{w}=0.1$ & $f_{w}=-0.1$ & $f_{w}=0.0$ & $f_{w}=0.1$ \\
\hline \multirow{8}{*}{1.0} & \multirow{4}{*}{0.1} & 0.0 & -1.594710 & -2.175171 & -2.891972 & -2.642449 & 3.515657 & -4.775250 & -3.533790 & -4.870410 & -7.408976 \\
\hline & & 0.2 & -1.401533 & -1.916397 & -2.544558 & -2.339826 & -3.103672 & -4.190680 & -3.136838 & -4.297609 & -6.471589 \\
\hline & & 0.4 & -1.256667 & -1.723788 & -2.287883 & -2.114304 & -2.798674 & -3.760927 & -2.840922 & -3.873808 & -5.784683 \\
\hline & & 0.6 & -1.142825 & -1.573377 & -2.088911 & -1.938122 & -2.561950 & -3.429562 & -2.609868 & -3.545184 & -5.256664 \\
\hline & 0.0 & \multirow{4}{*}{0.1} & -1.510673 & -2.069912 & -2.758452 & -2.525784 & -3.363229 & -4.567401 & -3.387321 & -4.665876 & -7.090334 \\
\hline & 0.2 & & -1.470402 & -2.000814 & -2.649451 & -2.432208 & -3.222360 & -4.349612 & -3.251530 & -4.455171 & -6.711020 \\
\hline & 0.4 & & -1.430128 & -1.931708 & -2.540434 & -2.338616 & -3.081466 & -4.131787 & -3.115710 & -4.244425 & -6.331641 \\
\hline & 0.8 & & -1.349570 & -1.793471 & -2.322351 & -2.151385 & -2.799603 & -3.696029 & -2.843991 & -3.822809 & -5.572702 \\
\hline 1.0 & \multirow{4}{*}{0.1} & \multirow{4}{*}{0.1} & -1.490538 & -2.035364 & -2.703953 & -2.478998 & -3.292798 & -4.458511 & -3.319429 & -4.560529 & -6.900684 \\
\hline 3.0 & & & -2.768508 & -3.729278 & -5.036390 & -4.354695 & -5.953586 & -8.514025 & -5.712118 & -8.275150 & -13.74434 \\
\hline 5.0 & & & -3.575755 & -4.863968 & -6.703440 & -5.555696 & -7.778276 & -11.54963 & -7.206194 & -10.82880 & -19.10840 \\
\hline 10.0 & & & -4.990203 & -6.942968 & -9.933557 & -7.614863 & -11.12871 & -17.66787 & -9.665832 & -15.51334 & -30.43573 \\
\hline
\end{tabular}




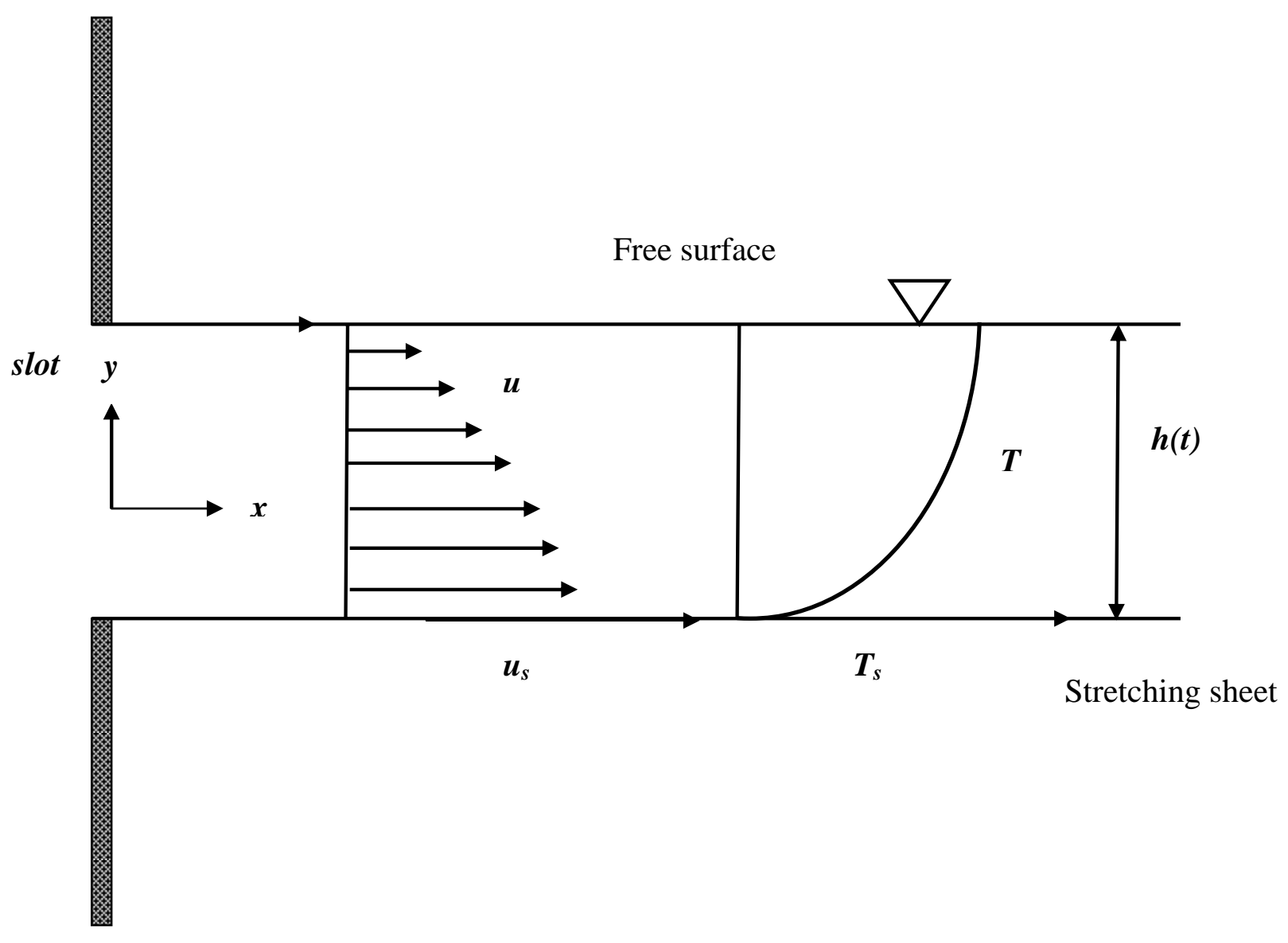

Fig.1: Schematic of a liquid film on an unsteady stretching sheet 


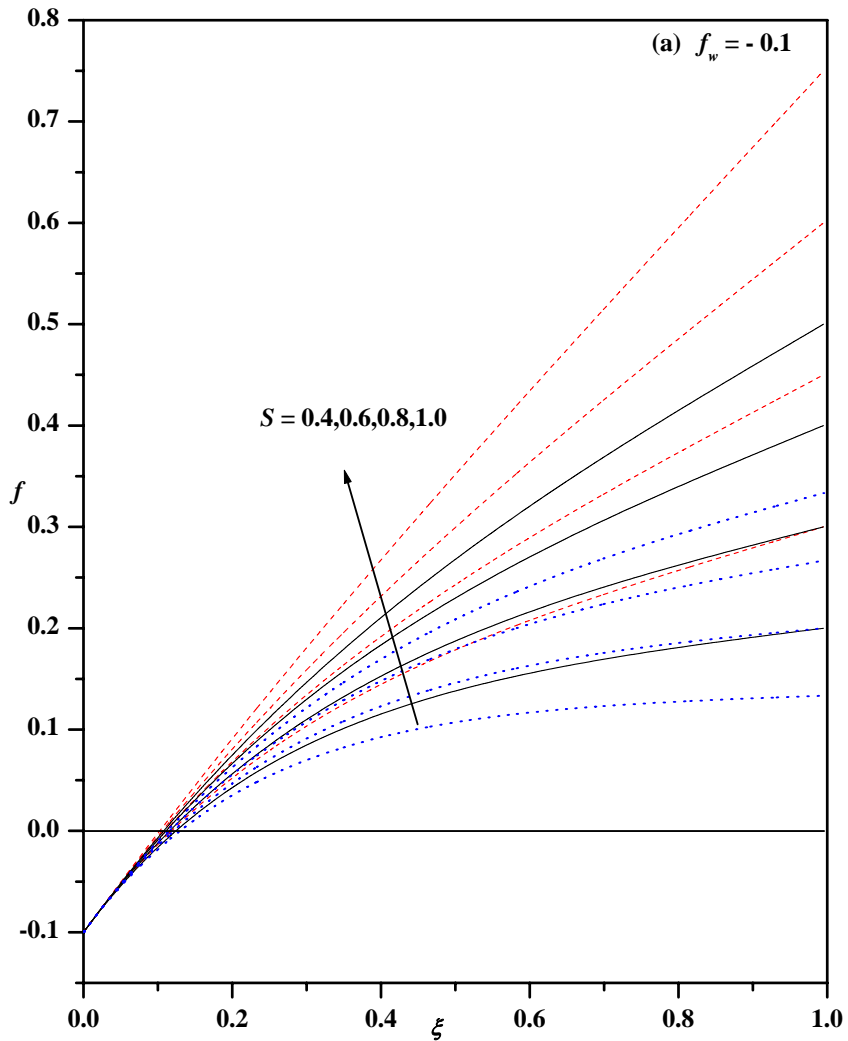

Fig.2(a): Transverse velocity profiles for different values of $n$ and $s$ with $f_{w}=-0.1$.

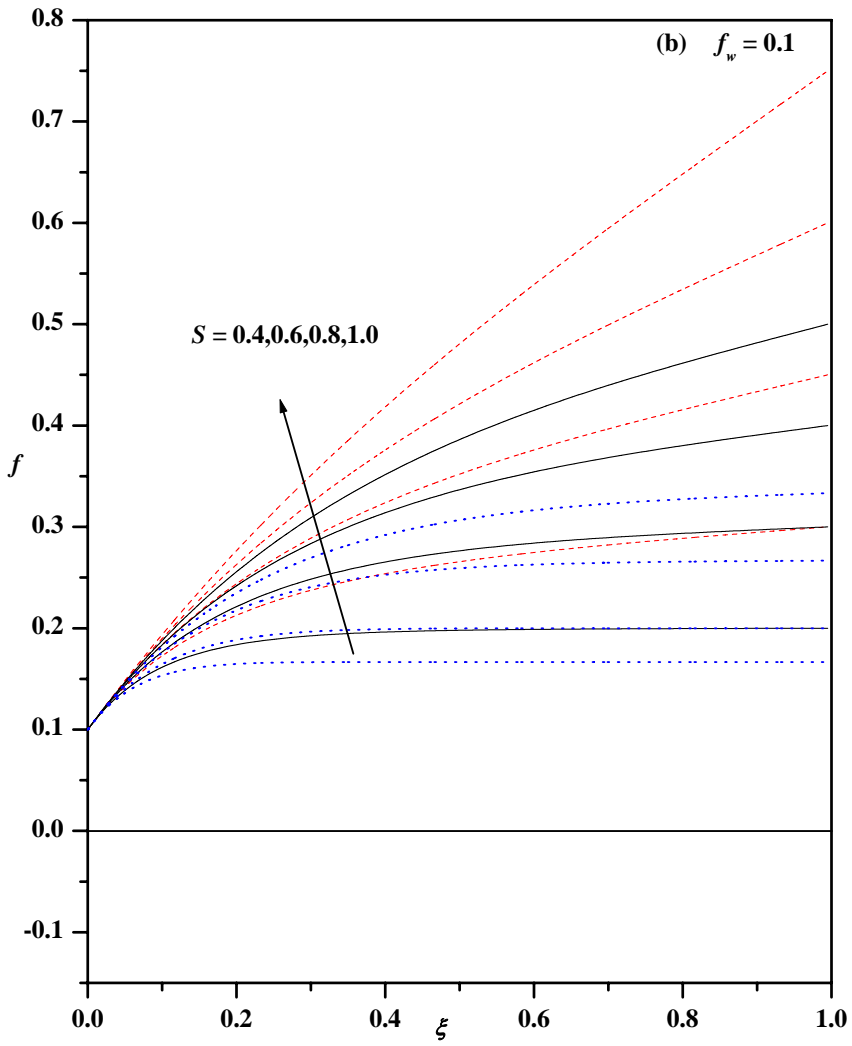

Fig.2(b): Transverse velocity profiles for different values of $n$ and $s$ with $f_{w}=0.1$.

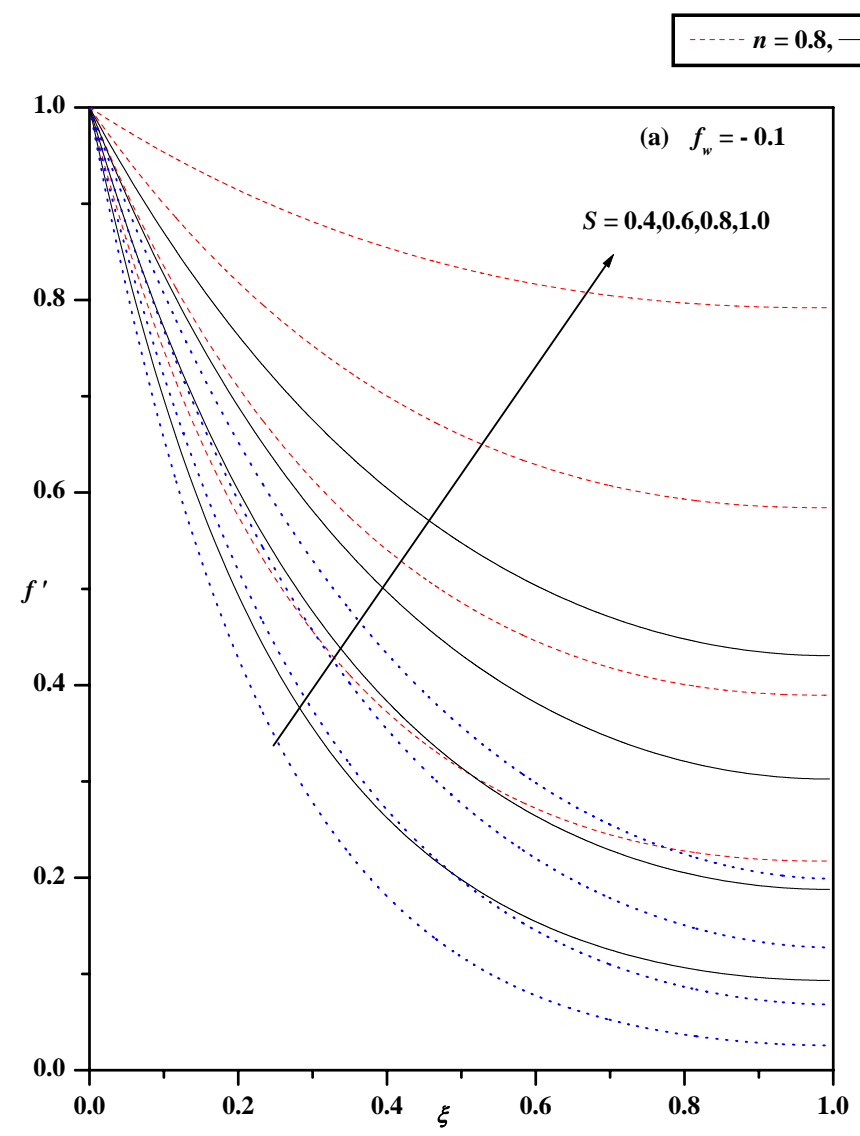

Fig.3(a): Horizontal velocity profiles for different values of $n$ and $s$ with $f_{w}=-0.1$.

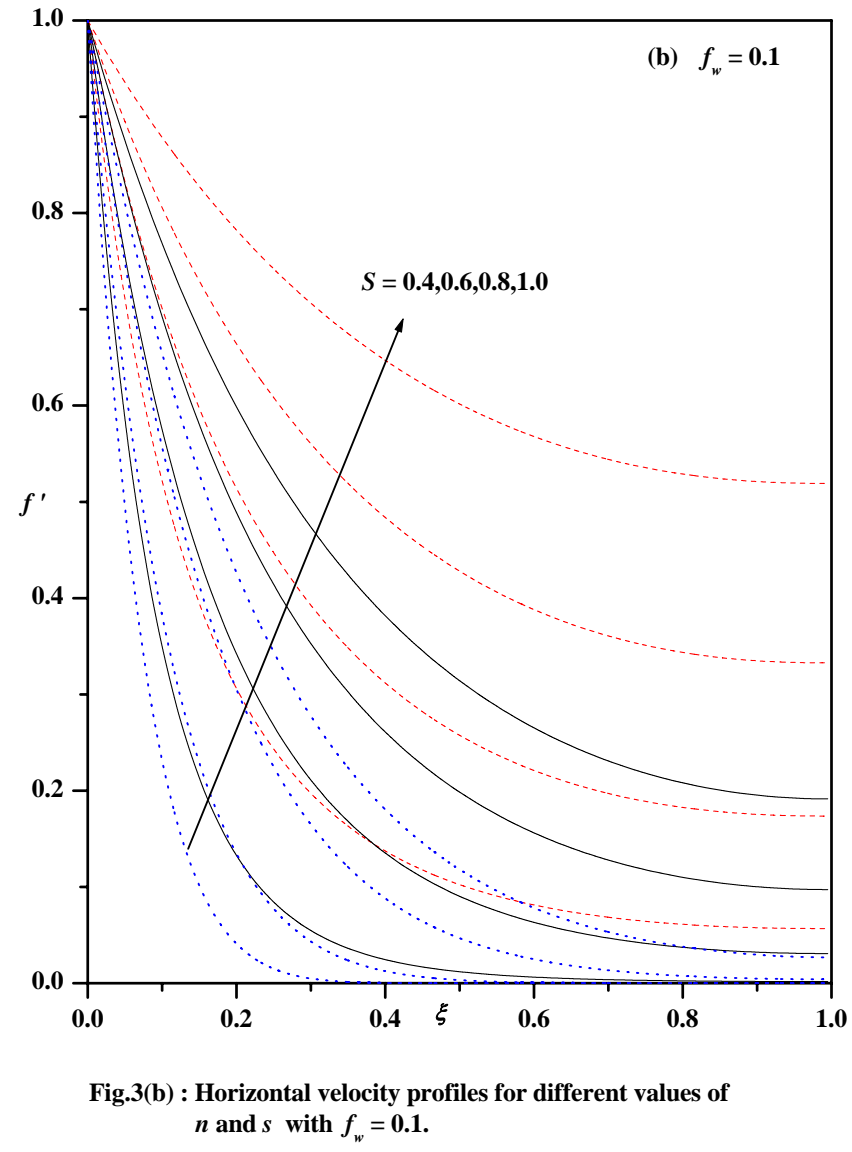




$$
n=0.8,-n=1.0, \cdots \cdots n=1.2
$$

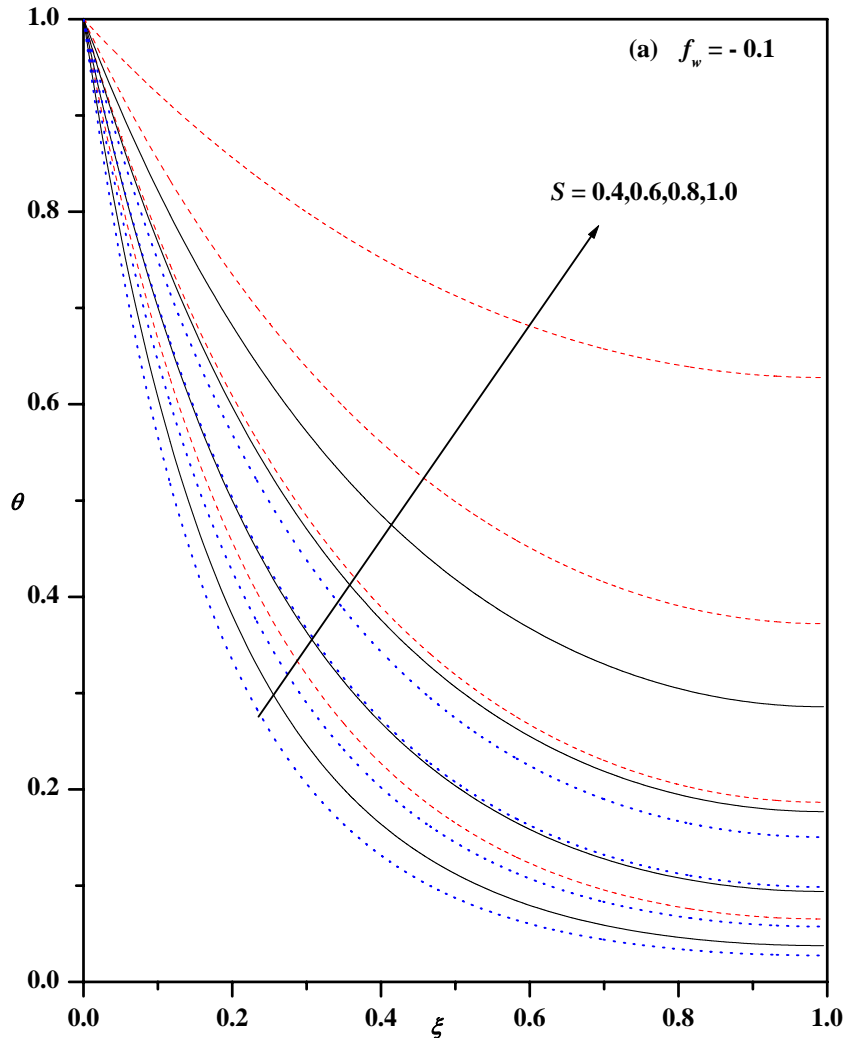

Fig.4(a): Temperature profiles for different values of $\boldsymbol{n}$ and $\boldsymbol{s}$ with $\operatorname{Pr}=1.0, E c=0.0, \varepsilon=0.0, f_{w}=-0.1$.

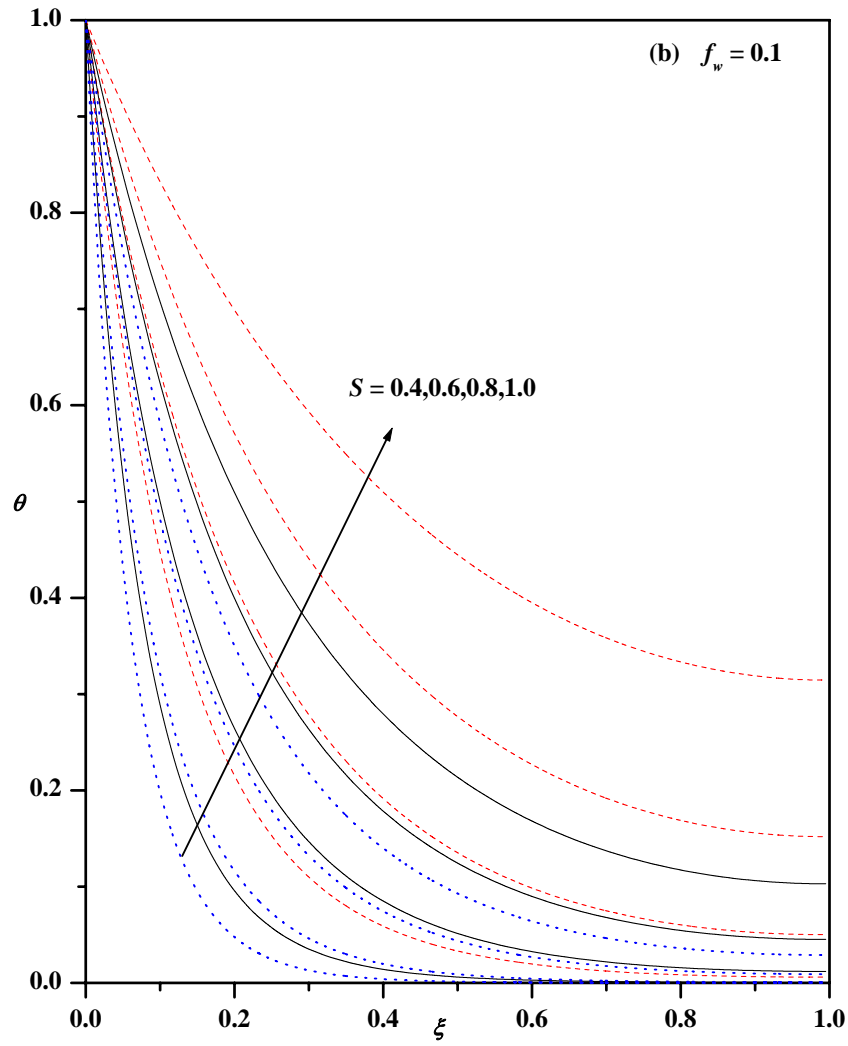

Fig.4(b): Temperature profiles for different values of $n$ and $s$ with $E c=0.0, \varepsilon=0.0, \operatorname{Pr}=1.0, f_{w}=0.1$.

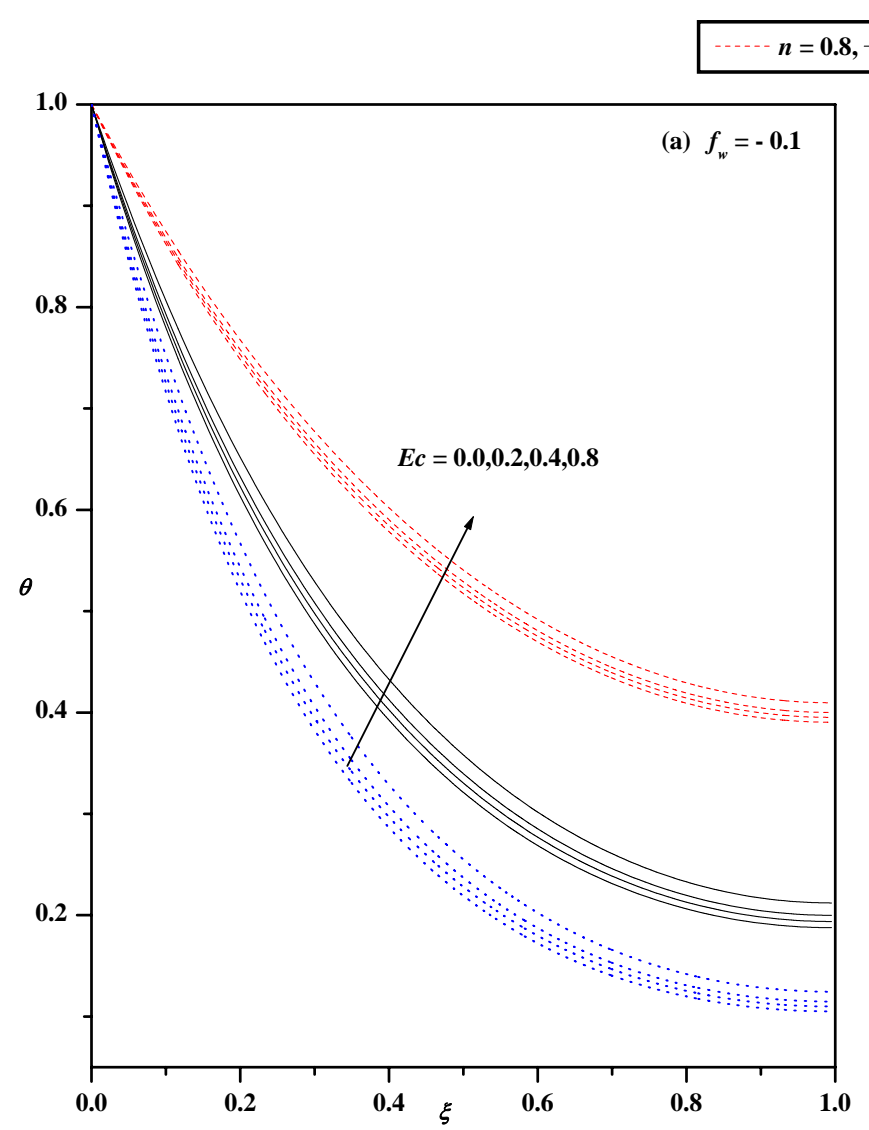

Fig.5(a): Temperature profiles for different values of $\boldsymbol{n}$ and $\boldsymbol{E c}$ with $\operatorname{Pr}=1.0, \varepsilon=0.1, s=0.8, f_{w}=-0.1$.

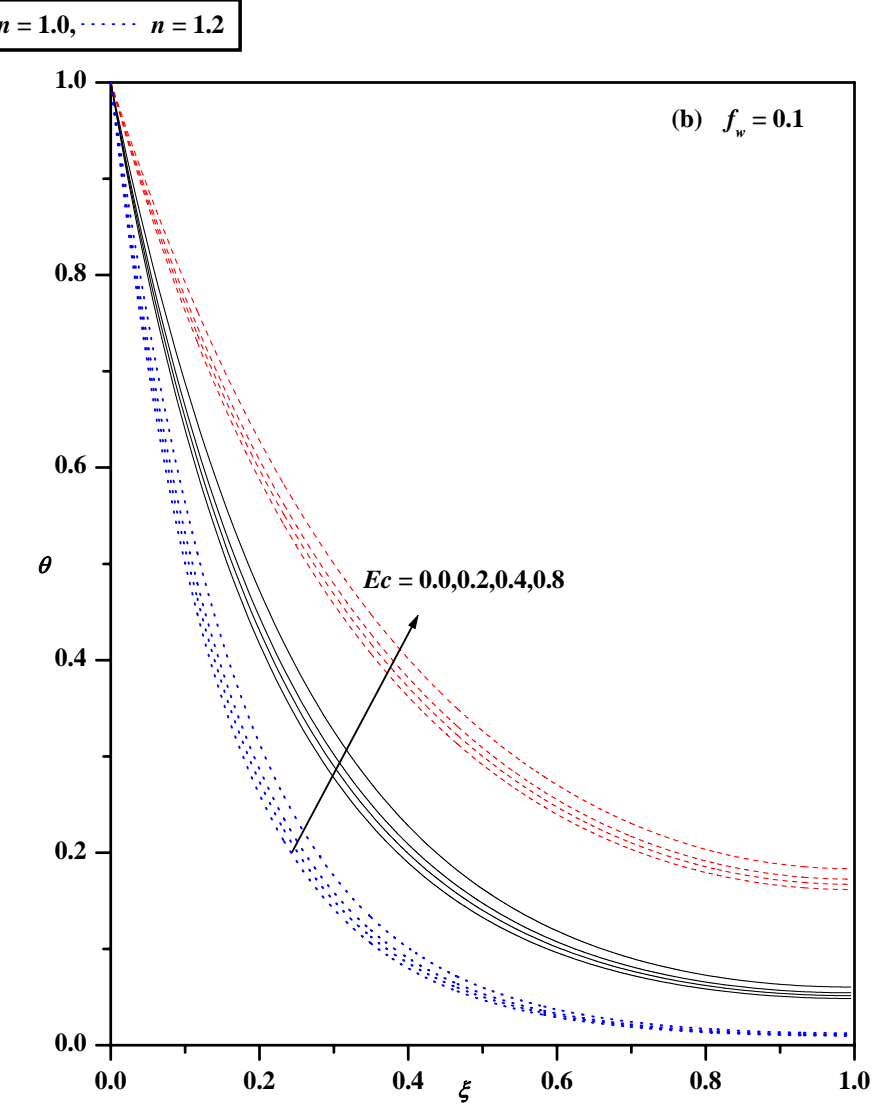

Fig.5(b): Temperature profiles for different values of $\boldsymbol{n}$ and $E c$ with $\operatorname{Pr}=1.0, \varepsilon=0.1, s=0.8, f_{w}=0.1$. 
$n=0.8,-n=1.0, \cdots \cdots n=1.2$

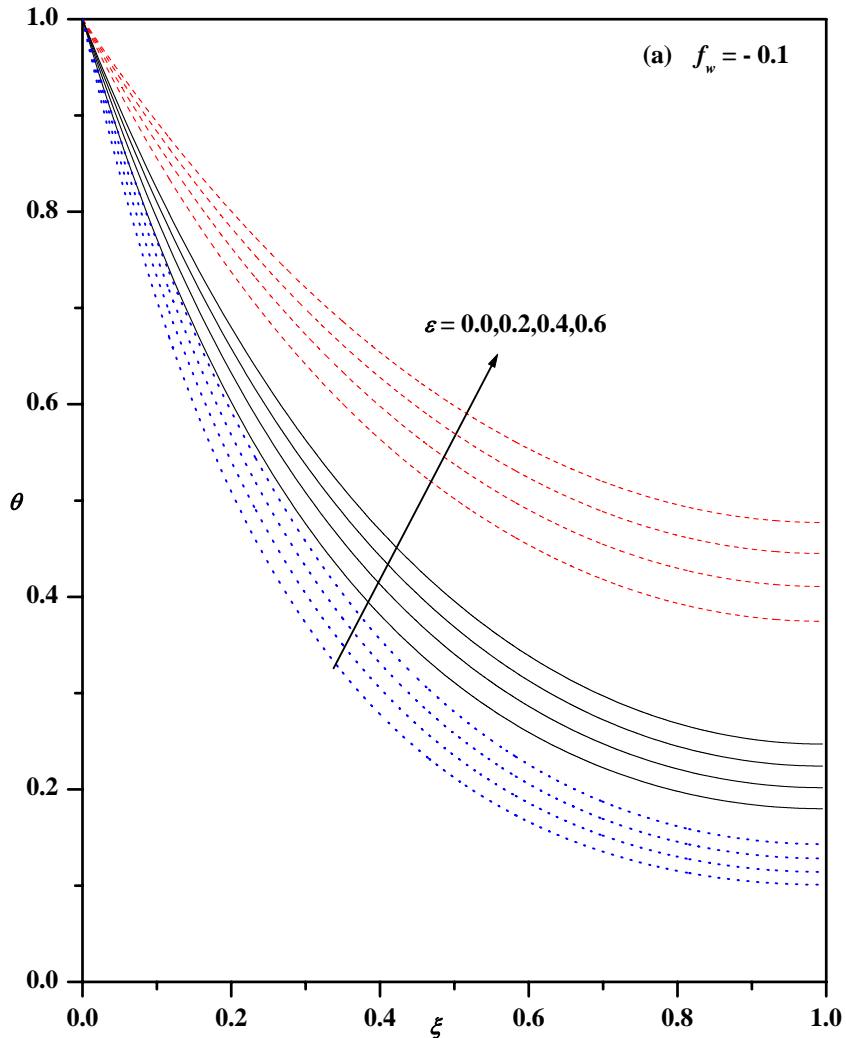

Fig.6(a): Temperature profiles for different values of $\boldsymbol{n}$ and $\varepsilon$ with $\operatorname{Pr}=1.0, E c=0.1, S=0.8, f_{w}=-0.1$.

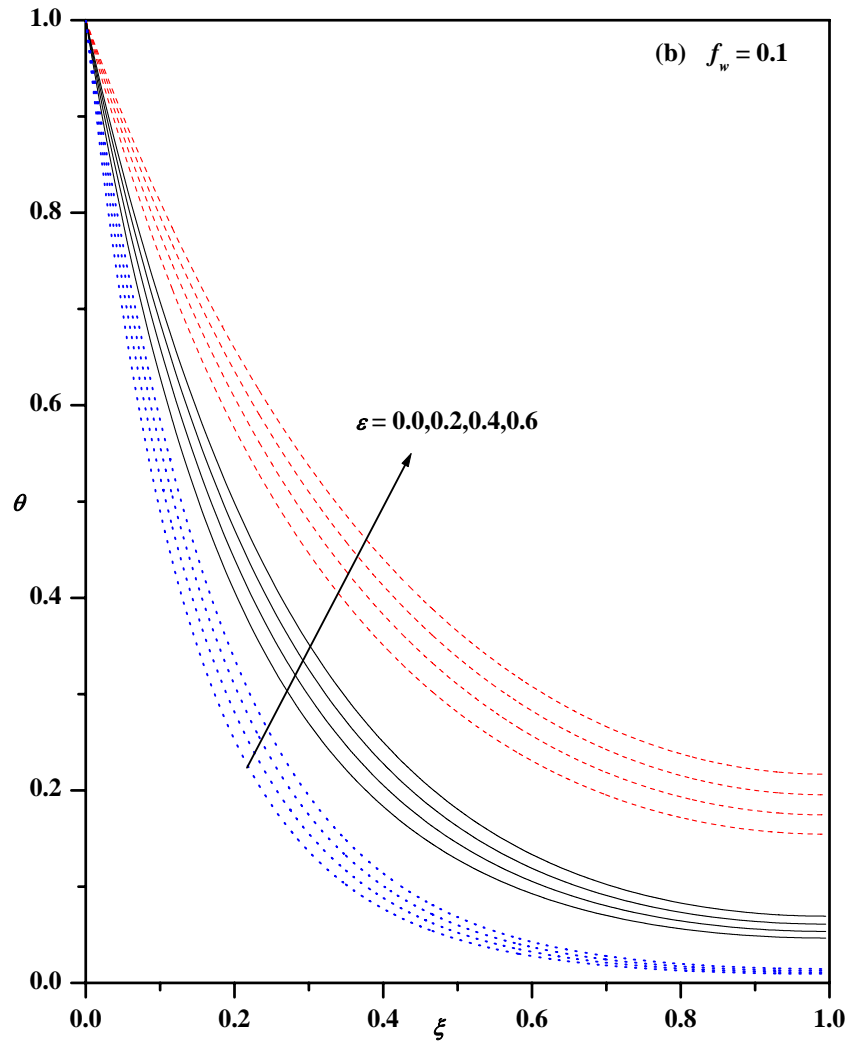

Fig.6(b): Temperature profiles for different values of $\boldsymbol{n}$ and $\varepsilon$ with $\operatorname{Pr}=1.0, E c=0.1, S=0.8, f_{w}=0.1$.

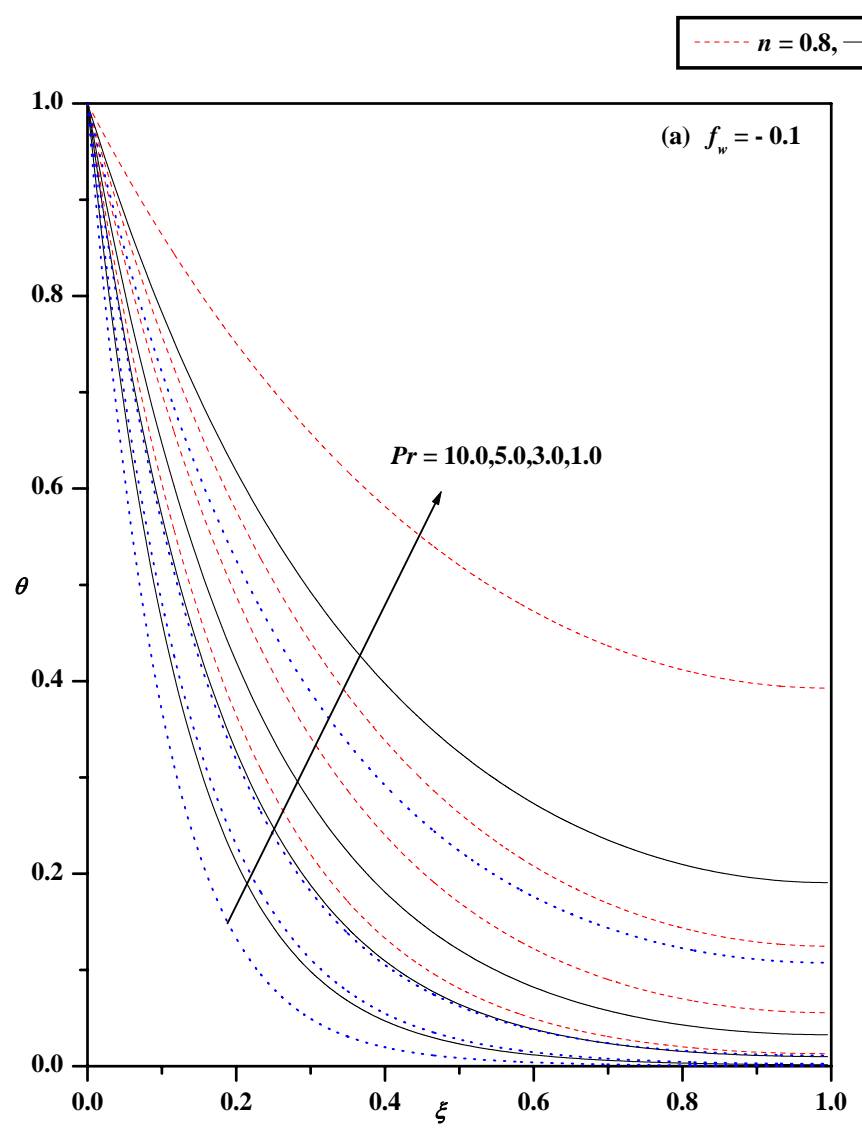

Fig.7(a): Temperature profiles for different values of $\boldsymbol{n}$ and $\mathrm{Pr}$ with $\varepsilon=0.1, E c=0.1, S=0.8, f_{w}=-0.1$.

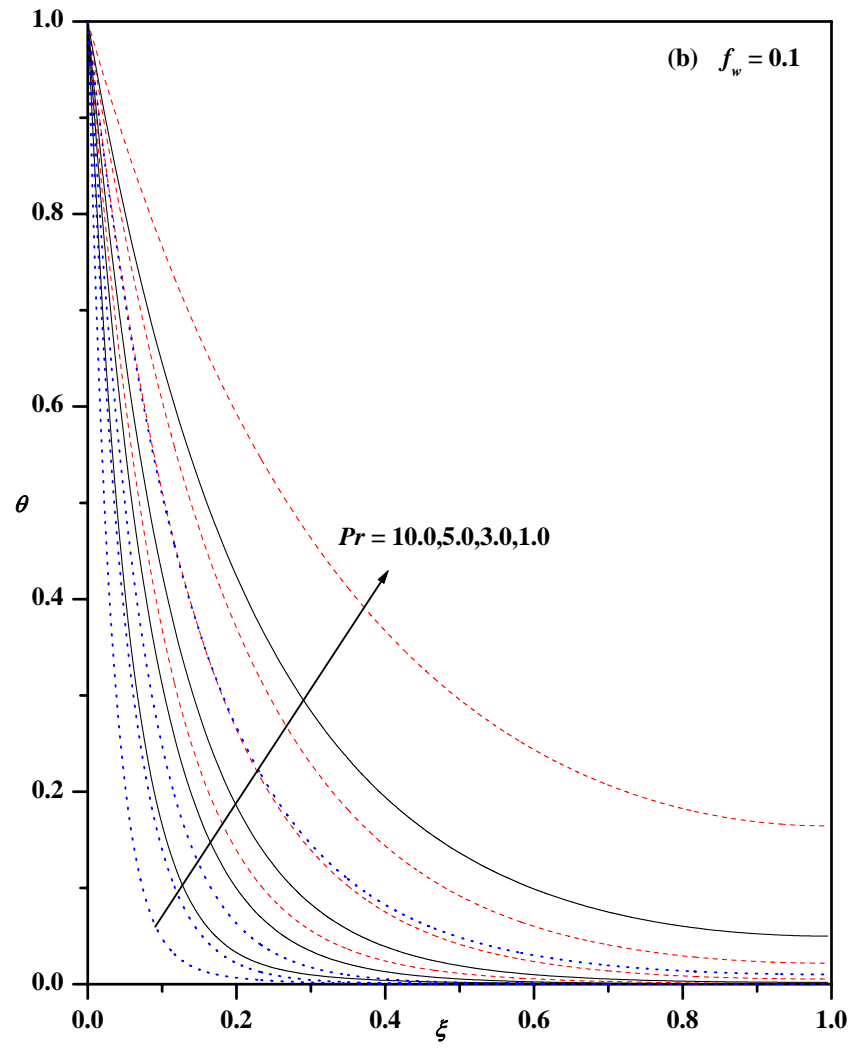

Fig.7(b): Temperature profiles for different values of $\boldsymbol{n}$ and $\mathrm{Pr}$ with $\varepsilon=0.1, E c=0.1, S=0.8, f_{w}=0.1$. 